\title{
Large-scale dust jets in the coma of 67P/Churyumov-Gerasimenko as seen by the OSIRIS instrument onboard Rosetta
}

\author{
L. M. Lara ${ }^{1}$, S. Lowry ${ }^{2}$, J.-B. Vincent ${ }^{3}$, P. J. Gutiérrez ${ }^{1}$, A. Rożek ${ }^{2}$, F. La Forgia ${ }^{4}$, N. Oklay ${ }^{3}$, H. Sierks ${ }^{3}$, C. Barbieri ${ }^{4,5}$, \\ P. L. Lamy ${ }^{6}$, R. Rodrigo ${ }^{7,8}$, D. Koschny ${ }^{9}$, H. Rickman ${ }^{10,16}$, H. U. Keller ${ }^{11}$, J. Agarwal ${ }^{3}$, A.-T. Auger ${ }^{6}$, M. F. A'Hearn ${ }^{12}$, \\ M. A. Barucci ${ }^{13}$, J.-L. Bertaux ${ }^{14}$, I. Bertini ${ }^{5}$, S. Besse ${ }^{9}$, D. Bodewits ${ }^{12}$, G. Cremonese ${ }^{15}$, B. Davidsson ${ }^{16}$, \\ V. Da Deppo ${ }^{17}$, S. Debei ${ }^{18}$, M. De Cecco ${ }^{19}$, M. R. El-Maarry ${ }^{20}$, F. Ferri ${ }^{5}$, S. Fornasier ${ }^{13}$, M. Fulle ${ }^{21}$, O. Groussin ${ }^{6}$, \\ P. Gutiérrez-Marques ${ }^{3}$, C. Güttler ${ }^{3}$, S. F. Hviid ${ }^{22}$, W.-H. Ip ${ }^{23}$, L. Jorda ${ }^{6}$, J. Knollenberg ${ }^{22}$, G. Kovacs 3 , J.-R. Kramm ${ }^{3}$, \\ E. Kührt ${ }^{22}$, M. Küppers ${ }^{24}$, M. Lazzarin ${ }^{4}$, Z.-Y. Lin ${ }^{23}$, J. J. López-Moreno ${ }^{1}$, S. Magrin ${ }^{4}$, F. Marzari ${ }^{4}$, H. Michalik ${ }^{11}$, \\ R. Moissl-Fraund ${ }^{24}$, F. Moreno ${ }^{1}$, S. Mottola ${ }^{22}$, G. Naletto ${ }^{5,17,25}$, M. Pajola ${ }^{5}$, A. Pommerol ${ }^{20}$, N. Thomas ${ }^{20}$, \\ M. D. Sabau ${ }^{26}$, and C. Tubiana ${ }^{3}$ \\ (Affiliations can be found after the references)
}

Received 16 March 2015 / Accepted 23 July 2015

\begin{abstract}
Context. During the most recent perihelion passage in 2009 of comet 67P/Churyumov-Gerasimenko (67P), ground-based observations showed an anisotropic dust coma where jet-like features were detected at $\sim 1.3 \mathrm{AU}$ from the Sun. The current perihelion passage is exceptional as the Rosetta spacecraft is monitoring the nucleus activity since March 2014, when a clear dust coma was already surrounding the nucleus at 4.3 AU from the Sun. Subsequently, the OSIRIS camera also witnessed an outburst in activity between April 27 and 30, and since mid-July, the dust coma at $r_{\mathrm{h}} \sim 3.7-3.6 \mathrm{AU}$ preperihelion is clearly non-isotropic, pointing to the existence of dust jet-like features.

Aims. We aim to ascertain on the nucleus surface the origin of the dust jet-like features detected as early as in mid-July 2014 . This will help to establish how the localized comet nucleus activity compares with that seen in previous apparitions and will also help following its evolution as the comet approaches its perihelion, at which phase most of the jets were detected from ground-based observations. Determining these areas also allows locating them in regions on the nucleus with spectroscopic or geomorphological distinct characteristics.

Methods. Three series of dust images of comet 67P obtained with the Wide Angle Camera (WAC) of the OSIRIS instrument onboard the Rosetta spacecraft were processed with different enhancement techniques. This was made to clearly show the existence of jet-like features in the dust coma, whose appearance toward the observer changed as a result of the rotation of the comet nucleus and of the changing observing geometry from the spacecraft. The position angles of these features in the coma together with information on the observing geometry, nucleus shape, and rotation, allowed us to determine the most likely locations on the nucleus surface where the jets originate from.

Results. Geometrical tracing of jet sources indicates that the activity of the nucleus of 67P gave rise during July and August 2014 to large-scale jet-like features from the Hapi, Hathor, Anuket, and Aten regions, confirming that active regions may be present on the nucleus localized at $60^{\circ}$ northern latitude as deduced from previous comet apparitions. There are also hints that large-scale jets observed from the ground are possibly composed, at their place of origin on the nucleus surface, of numerous small-scale features.
\end{abstract}

Key words. comets: general - comets: individual: 67P/Churyumov-Gerasimenko

\section{Introduction}

After the discovery of comet $67 \mathrm{P} /$ Churyumov-Gerasimenko (hereafter 67P) in 1969, it has been seen from ground-based observatories at seven apparitions, and it is currently being monitored by the European Space Agency's Rosetta spacecraft since March 2014.

Optical observations at $r_{\mathrm{h}} \approx 2.3$ AU postperihelion in the 2003 passage showed a clear dust coma extending $20000 \mathrm{~km}$ from the comet (Weiler et al. 2004) as well as a long neck-line structure due to large dust particles residing in the comet orbital plane that were most likely ejected at $r_{\mathrm{h}} \approx 3 \mathrm{AU}$ pre-perihelion (Fulle et al. 2004; Moreno et al. 2004). Vincent et al. (2013) analyzed ground-based images acquired in the pre-perihelion phase of 2008 and 2009. These images did not display unambiguous coma structures until about a month before perihelion. However, the isophotes presented several distortions in addition to an extension in the tail direction, which were enhanced by the Laplace filtering (Boehnhardt \& Birkle 1994). As Vincent et al. (2013) did not detect these patterns with other filters such as Larson-Sekanina (rotational-difference) (Larson \& Sekanina 1984) or radial renormalization (A'Hearn et al. 1986), they were considered artifacts due to the filtering. Moreover, their signal level may have been too close to the background for them to be enhanced by traditional filters.

During the 2009 passage, Lara et al. (2011) studied the comet activity around perihelion ( $r_{\mathrm{h}}$ ranging from 1.314 to 1.269 AU) when the equatorial and southern latitudes of the comet nucleus received most of the insolation. The morphological analysis of the dust coma revealed that structures do indeed exist. In addition to the dust tail in the southwestern direction, four more small-scale structures were detected by using multiple methods. These structures were also confirmed by the distortion of the isophotes at the same position angles $(\alpha)$ where 
the structures were found by different enhancement techniques. During January and February 2009, a faint structure was seen at $\alpha \approx 330^{\circ}$, which feature seemed to have disappeared after perihelion. Additionally, another broad structure at $\alpha \approx 45^{\circ}$ seemed to split into two narrower features on February 26, 2009, to become a considerably fainter one at $\alpha \approx 75^{\circ}$ during March 2009.

By using the same data set as Lara et al. (2011), Vincent et al. (2013) obtained an estimate of the spin axis rotation that has been confirmed by Rosetta spacecraft data (Mottola et al. 2014; Sierks et al. 2015). They also localized the most prominent active areas on the surface of the nucleus during the nucleus season it was observed. Around perihelion, the activity seen in the ground-based images could be reproduced with three active regions localized at $-45^{\circ}, 0^{\circ}$ and $+60^{\circ}$ latitude. These results on the location of confined activity were also valid for the 2003 passage. For the 2015 perihelion passage, Vincent et al. (2013) predicted that if the comet behaved as in 2003 and 2009, the main jets should become visible from Earth a month before perihelion, that is, mid-July 2015. They also predicted that the activity would mainly take place at the equator and mid-latitudes around perihelion, with an emphasis on the hemisphere illuminated around perihelion. We note that the model of Vincent et al. (2013) reproduces the largest sources of activity while not ruling out that there might be many other small sources of activity distributed all over the surface, which cannot be directly detected from ground-based observations. As these authors noted, it was also very likely than some of the jets studied were not single features, but the result of a number of small jets in a given region, whose emissions are seen as coming from a single large source.

The images acquired by the OSIRIS camera (Keller et al. 2007) beginning in mid-July, when 67P was at 3.6 AU from the Sun, started to show a well-developed dust coma whose isophotes were clearly distorted, a straightforward indication of non-isotropic dust emission. At that heliocentric distance, and given the location of the spin axis, the illuminated areas on the nucleus correspond to the northern hemisphere.

The analysis of three OSIRIS data sets pertaining to July 20-21, 25-26 and August 2, 2014, allows us to determine where the dust jet-like features seen in the images originate on the nucleus for (i) a comparison with those that produced the jets seen around the 2003 and 2009 perihelion; (ii) establishing a pattern of the jet activity throughout all stages of the mission; and (iii) linking the small-scale jets seen when the nucleus has been spatially resolved (Vincent et al. 2015) by the OSIRIS cameras with those seen from the ground.

Unfortunately, ground-based observations of the comet at similar pre-perihelion distances as those analyzed in this work cannot provide any information on dust coma anisotropies because the signal-to-noise ratio $(\mathrm{S} / \mathrm{N})$ in the images (C. Snodgrass, priv. comm.) and the spatial resolution are too low for a meaningful comparison with the results derived in this work.

\section{Observations and detection of large-scale jets from image processing}

To derive the position of the active zones on the nucleus by analyzing the dust structures that are clearly seen in the coma, we selected images of 67P that were acquired in July and August 2014 with the OSIRIS Wide Angle Camera (WAC) with the Vis610 filter $(\lambda / \Delta \lambda=616.6 / 9.84 \mathrm{~nm})$ from July 20 20:39 UT to July 21 08:34 UT (i.e., one nucleus rotation), July 25, 20:50 UT to July $2606.32 \mathrm{UT}$, covering approximately $10 \mathrm{~h}$ of comet nucleus rotation, and August 2 00:24 UT spanning $17 \mathrm{~h}, \sim 1.5$ rotations of the nucleus. Table 1 lists the date, UT, as well as the heliocentric and spacecraft-comet distances, and the spatial scale (meters per pixel).

The jet-like features were enhanced by making use of the radial renormalization technique (A'Hearn et al. 1986) and the Laplace filtering method (Boehnhardt \& Birkle 1994) on every image studied in this work. Each feature detected in the Laplacefiltered images is confirmed by the radial renormalized and by unsharp masked images. The latter two are enhancement techniques that are less prone to artifacts (Samarasinha \& Larson 2014). Figures 1-3 show the dust coma of 67P on July 20-21, 25-26 and August 2, 2014 after applying enhancement techniques to clearly see the jet-like features in its coma. We underline that the required overexposure of the nucleus to detect faint coma structures gives rise to several ghosts (the bright circular area to the right of the nucleus positions).

For images acquired on July 20-21 and 25-26, 2014, the comet nucleus was already spatially resolved. However, we were still able to use the radial renormalization technique without introducing many artifacts in the innermost coma (i.e., at the comet limb) (see Figs. 1 and 2). The enhanced images were converted into polar coordinates $(\rho, \alpha)$, and we extracted the emission at selected cometocentric distances $\rho$ for angular positions $\alpha$ ranging from $0^{\circ}$ to $360^{\circ}$ to determine where a jet-like feature was unequivocally detected. This procedure was done for the 25 images in the July 20-21 and 25-26 series, and the results were crosschecked with features found in the Laplace-filtered (Boehnhardt \& Birkle 1994) images (see Fig. 2).

For August 2, 2014, the comet nucleus is very well resolved, occupying a large portion of the field of view (FoV) of the windowed $(512 \times 512$ pixels $)$ WAC detector.

The bright limb shape of the nucleus on August 2 creates artifacts in the few innermost pixels when producing azimuthally averaged profiles that are used to subtract from the observed image. Their appearance as broad angular features makes it difficult to accurately determine the angular position of the jet (see Fig. 3). Thus, we made use of the Laplace-filtered images (Boehnhardt \& Birkle 1994) with different filter widths to crosscheck that the detected features are present regardless of the filter width. We also verified that the enhanced jet-like features in Laplace-filtered images were found when applying the radial renormalization method. The Laplace-filtered images were converted into polar coordinates after centering them at the nucleus coordinates at the date and time of observation provided by the Spice kernels. After this, the procedure was the same as stated above for the July series.

From a general point of view, these enhancement techniques have permitted us to detect one jet-like feature in images obtained on July 20-21, up to three in images from July 25-26 and a maximum of four on August 2. For some images in the July 25-26 and August 02 series, a very broad feature, slightly detached from the nucleus surface, is also clearly detected. To be precise, it is detected on July 25 at $23: 25 \mathrm{UT}$ at PA $\sim 185^{\circ}$, on August 2 at 02:24 and 03:24 UT (not shown in Fig. 3), or a shelllike feature on July 26 at 06:32 UT covering PA from $\sim 110^{\circ}$ to $\sim 160^{\circ}$. The obtained position angles for every data set are listed in Table 1.

Lin et al. (2015) have also studied the morphology and dynamics of the jet-like features found in the coma of $67 \mathrm{P}$ on time series covering a full rotation of the nucleus on August 16, September 16-17 and 27, 2014. At that time, the Rosetta cometocentric distance ranged from 98.7 to $106.1 \mathrm{~km}$, allowing for a high spatial resolution of nucleus and jet. The jets shown 
Table 1. Observations log.

\begin{tabular}{|c|c|c|c|c|c|c|c|c|}
\hline Date & UT & $r_{\mathrm{h}}(\mathrm{AU})$ & $d(\mathrm{~km})$ & (m/pix) & $\bar{~} \overline{\alpha_{1}\left({ }^{\circ}\right)}$ & $\overline{\alpha_{2}\left({ }^{\circ}\right)}$ & $\overline{\alpha_{3}\left({ }^{\circ}\right)}$ & $\bar{~} \overline{\alpha_{4}\left({ }^{\circ}\right)}$ \\
\hline $2014-07-20$ & $20: 39$ & 3.69 & 5473 & 568 & 108 & & & \\
\hline 2014-07-20 & $21: 27$ & 3.69 & 5450 & 565 & 107 & & & \\
\hline 2014-07-20 & $22: 15$ & 3.69 & 5426 & 563 & 110 & & & \\
\hline 2014-07-20 & 23:02 & 3.69 & 5403 & 561 & 118 & & & \\
\hline 2014-07-20 & $23: 50$ & 3.69 & 5380 & 559 & 109 & & & \\
\hline 2014-07-21 & $00: 38$ & 3.69 & 5357 & 556 & 90 & & & \\
\hline 2014-07-21 & $01: 25$ & 3.69 & 5334 & 553 & 62 & & & \\
\hline 2014-07-21 & $02: 13$ & 3.69 & 5311 & 551 & 68 & & & \\
\hline 2014-07-21 & $03: 48$ & 3.69 & 5265 & 546 & 137 & & & \\
\hline 2014-07-21 & $05: 24$ & 3.69 & 5218 & 541 & 140 & & & \\
\hline 2014-07-21 & $06: 59$ & 3.69 & 5172 & 537 & 134 & & & \\
\hline 2014-07-21 & 08:34 & 3.69 & 5126 & 532 & 98 & & & \\
\hline $2014-07-25$ & $20: 50$ & 3.65 & 2836 & 286 & 117 & 150 & & \\
\hline 2014-07-25 & $23: 25$ & 3.65 & 2804 & 283 & 160 & $194-220$ & & \\
\hline 2014-07-26 & 00:04 & 3.65 & 2795 & 282 & 156 & & & \\
\hline 2014-07-26 & $00: 43$ & 3.65 & 2787 & 281 & 161 & & & \\
\hline 2014-07-26 & 01:22 & 3.65 & 2780 & 281 & 156 & & & \\
\hline 2014-07-26 & 02:00 & 3.65 & 2771 & 280 & 195 & & & \\
\hline $2014-07-26$ & $02: 39$ & 3.65 & 2763 & 279 & 192 & & & \\
\hline 2014-07-26 & 03:18 & 3.65 & 2755 & 278 & $87^{a}$ & 186 & & \\
\hline 2014-07-26 & 03:57 & 3.65 & 2748 & 278 & $95^{a}$ & $182^{a}$ & & \\
\hline 2014-07-26 & 04:35 & 3.65 & 2739 & 277 & $147^{a}$ & $222^{a}$ & 351 & \\
\hline 2014-07-26 & 05:14 & 3.65 & 2731 & 276 & 158 & $221^{a}$ & & \\
\hline 2014-07-26 & 05:53 & 3.65 & 2723 & 275 & 146 & 235 & 338 & \\
\hline 2014-07-26 & $06: 32$ & 3.65 & 2715 & 274 & 238 & 339 & & \\
\hline 2014-08-02 & $00: 24$ & 3.62 & 716 & 72 & 320 & 337 & 345 & \\
\hline 2014-08-02 & $01: 24$ & 3.62 & 704 & 71 & 5 & 329 & & \\
\hline 2014-08-02 & $02: 24$ & 3.62 & 692 & 70 & 316 & 318 & & \\
\hline 2014-08-02 & 03:24 & 3.62 & 680 & 69 & 319 & & & \\
\hline 2014-08-02 & $06: 32$ & 3.62 & 643 & 65 & $313-342$ & & & \\
\hline 2014-08-02 & 08:24 & 3.62 & 621 & 63 & 19 & 37 & & \\
\hline 2014-08-02 & 09:24 & 3.62 & 609 & 62 & 13 & 32 & 52 & \\
\hline 2014-08-02 & $11: 24$ & 3.62 & 585 & 59 & 304 & 320 & 350 & \\
\hline 2014-08-02 & $12: 24$ & 3.62 & 574 & 58 & 318 & 323 & 338 & \\
\hline 2014-08-02 & $13: 24$ & 3.62 & 562 & 57 & 0 & 14 & 331 & 344 \\
\hline 2014-08-02 & $14: 24$ & 3.62 & 550 & 56 & 316 & 342 & & \\
\hline 2014-08-02 & $15: 24$ & 3.62 & 539 & 54 & 321 & & & \\
\hline 2014-08-02 & $16: 24$ & 3.62 & 527 & 53 & 328 & & & \\
\hline 2014-08-02 & $17: 24$ & 3.62 & 516 & 52 & 352 & & & \\
\hline
\end{tabular}

Notes. $r_{\mathrm{h}}$ refers to the comet heliocentric distance in $\mathrm{AU}, d$ is the Rosetta s/c-nucleus distance in $\mathrm{km}$, and $\alpha_{n}\left({ }^{\circ}\right)$ with $n$ going from 1 to 4 are the position angles at which jet-like features are detected measured from top in the images (Figs. 1-3) counterclockwise. ${ }^{(a)}$ Curved features.

in Fig. 1 in Lin et al. (2015, corresponding to observations on August 16) and those two weeks earlier (i.e., August 2) enhanced in Fig. 3 are remarkably similar in position angles, curvature, and appearance.

\section{Determining jet-source locations on the nucleus}

\subsection{Method 1: Geometrical tracing of local jet sources}

As seen in our observations, jet-like features are detected in most of the images and seem to rotate with the nucleus. Similar studies on other comets (Vincent et al. 2010; Farnham et al. 2013; Mueller et al. 2013; Lin et al. 2012) have shown that jets can often be associated with specific locations on the surface that present a different composition and morphology than other areas. For instance, some areas of comet 9P/Tempel 1 remained active and produced jets even far into the night, hinting at sublimation of material much more volatile than water ice (Vincent et al. 2010; Feaga et al. 2007). To understand which areas of comet 67P were active in July and August 2014, we performed a blind geometric inversion (Vincent et al. 2010) of the jet sources. The algorithm considers each feature in the images as an isolated jet. Although it seems obvious that jets can be tracked from one image to another, their appearance can be deceiving, and we did not impose this condition for the inversion. The inversion is then purely geometric: For each collimated structure, it is safe to assume that the dust is emitted in a plane defined by the observer (i.e., the OSIRIS camera onboard the Rosetta spacecraft) line of sight and the central line of the jet (position angle defined above). This is a very conservative approach; in reality, the jets probably have a tube or cone structure, not that of a plane. But we chose this approach to account for the fact that from one image alone we cannot decide whether the jet is pointing toward or away from us. In a second step we calculated the intersection between the jet-plane and a shape model of the nucleus, oriented beforehand to match the geometric conditions of our observations. We used the shape model reconstructed by Preusker et al. (2015), and all information on spacecraft and comet attitudes and trajectories was retrieved with the SPICE library (Acton 1996). Each jet-plane/comet intersection defines a line 

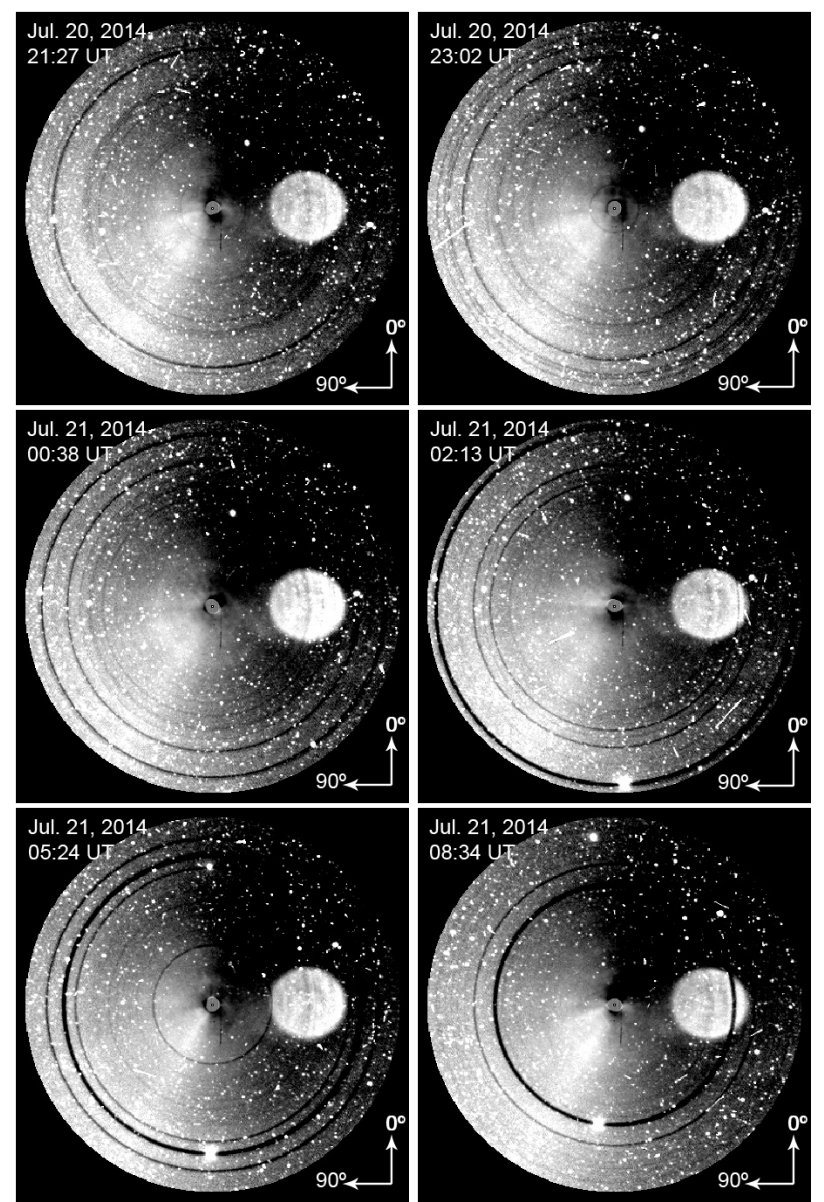

Fig. 1. Series of radially renormalized images of $67 \mathrm{P}$ acquired in the optical wavelength range $(616 \mathrm{~nm})$ with the OSIRIS WAC on July 20-21, 2014. Date and time of image acquisition is indicated on the frame, as well as the arrows, which indicate the angle convention for placing the detected jet-like features. The comet nucleus is at the center of the FoV, which ranges from $226 \times 226 \mathrm{~km}$ on July 20 at 21:27 UT to $212 \times 212 \mathrm{~km}$ on July 21 at 08:34 UT. and the spatial resolution is listed in Table 1 for each observation date. The artifact to the right of each frame is due to the ghost that is caused by overexposure of the nucleus. The look-up table is linear, white represents higher brightness intensity.

of possible sources on the surface of the nucleus. For the same jet observed at several different times, this technique provides a different set of sources; their intersection defines the location of the unique source. By repeating this process for each jet and each image, we can refine the inversion and build a probability map of source areas.

This inversion is as good as the resolution of our images. Here we were able to refine the source locations to areas of about some hundred meters on the surface. Applying this approach to the images acquired on July 20-21 and on August 2, 2014, we find that most jets arise from the north polar region Hapi defined in Sierks et al. (2015) and Thomas et al. (2015), which is the transition area between the two lobes. Figure 4 shows the results of this inversion for one of the acquired images. We note that for every image in that sequence (July 20-21, 2014), the results are the same in terms of jet-like feature sources. Hence, we find two distinct sources in the Hapi region, but it is very likely that the whole area is active. Both regions do not emit dust jets at the same time. We see a progression from East to West as the nucleus rotates and the area in the Hapi region receives more solar energy. From data acquired by the instruments onboard Rosetta

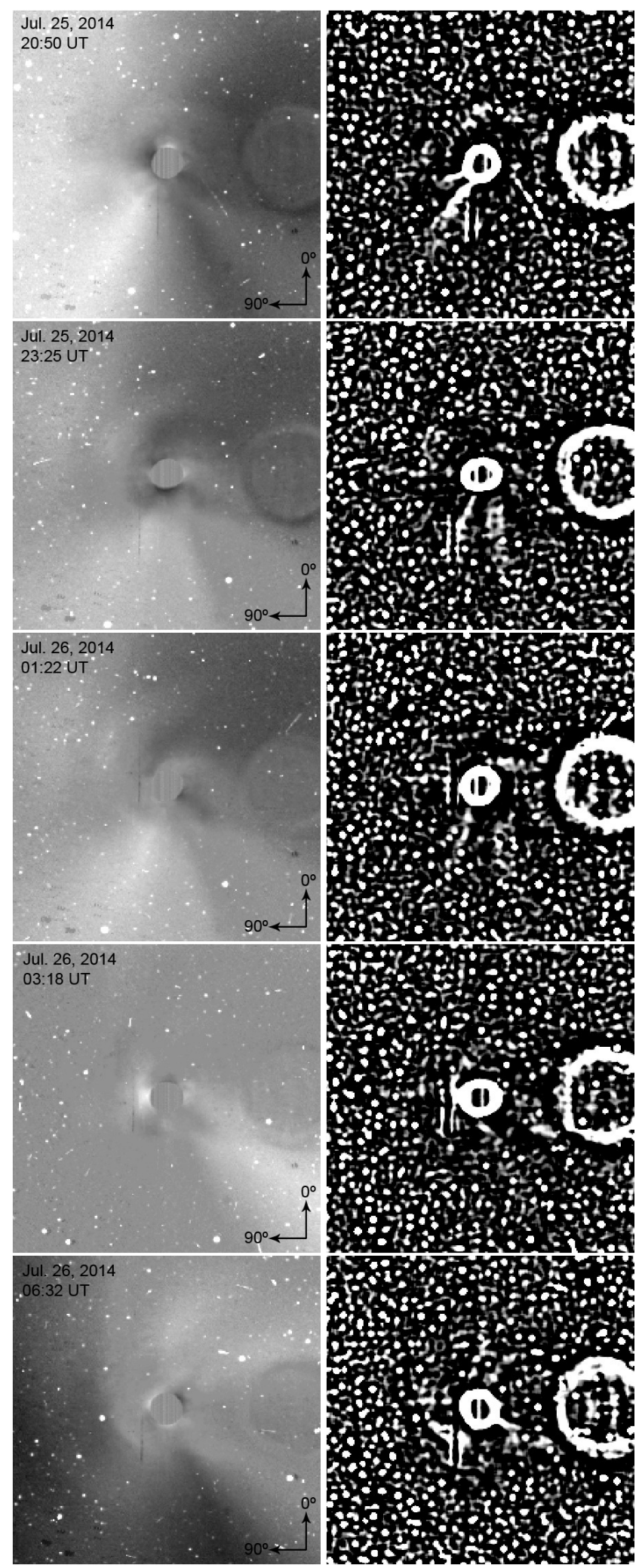

Fig. 2. Series of images of 67P acquired in the optical wavelength range $(616 \mathrm{~nm})$ with the OSIRIS WAC on July 25-26, 2014. The left panel shows the radially renormalized images, and the right one displays the corresponding Laplace-filtered image. Date and time of image acquisition is indicated on the frame, as well as the arrows, which indicate the angle convention for placing the detected jet-like features. The comet nucleus is at the center of the FoV, which is $73 \times 73 \mathrm{~km}$ at the comet, and the spatial resolution is $\approx 280 \mathrm{~m} / \mathrm{pix}$. The artifact to the right of each frame is due to the ghost that is caused by overexposure of the nucleus. The look-up table is linear, white represents higher brightness intensity. 

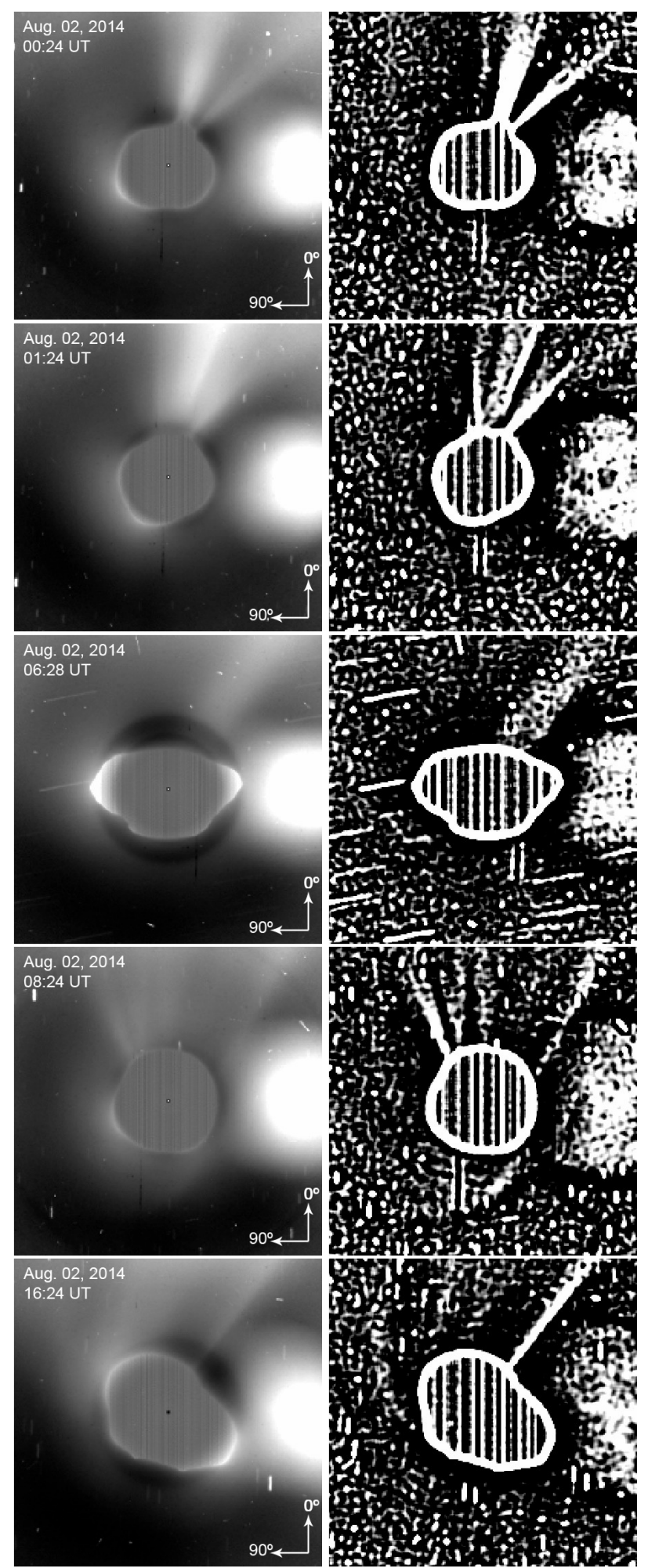

Fig. 3. Series of images of 67P acquired in the visible range $(616 \mathrm{~nm})$ with the OSIRIS WAC on August 02, 2014. The panels show at the left the radially renormalised images and to the right the corresponding Laplace filtered image. The jet-like features clearly appear as very confined filaments of material varying in angular width. The FoV ranges from $17.2 \times 17.2$ to $13.9 \times 13.9 \mathrm{~km}$ at the comet whereas the spatial resolution increases from $72 \mathrm{~m} /$ pix up to $52 \mathrm{~m} /$ pix. Date and time of image acquisition is on the frame, as well as the arrows indicating the angle convention for placing the detected jet-like features. The artifact to the right of each frame is due to the ghost resulting from overexposure of the nucleus. The look-up table is linear, white represents higher brightness intensity. until mid-October, Hässig et al. (2015) and Gulkis et al. (2015) have clearly established that the comet's global activity is driven by solar insolation. In the same figure, we show the solar elevation (in degrees) for each facet of the nucleus shape model by Preusker et al. (2015) averaged over the time frame covered by the images obtained on July 20-21, 2014.

The spatial resolution of this inversion does not allow us to link active sources and a specific morphologic feature, although the sources seem to align with the southern edge of Hapi, at the interface with Hathor. This region has been identified as being distinctly different from the rest of the nucleus, with a brighter albedo and a less red spectrum in the visible and infrared (Sierks et al. 2015; Capaccioni et al. 2015). Moreover, Keller et al. (2015) have calculated the variation of the insolation as a function of heliocentric distance and diurnal (spin dependent) variation, concluding that in its current orbit and rotation axis orientation, the northern and southern hemispheres of the nucleus of comet 67P suffer very different amounts of irradiation that can be used for sublimation of water ice. According to their calculation, for August 9, 2014, an enhanced erosion, compared to surrounding regions, of $\sim 1 \mathrm{~mm}$ takes place in the region along the transition from the valley bottom Hapi to the cliff wall Hathor during that day, indicating a higher activity localized in that area that most likely causes the jet-like structures seen in Figs. 1-3.

\subsection{Method 2: Geometrical tracing of jet sources using tolerance limits}

We employed a second approach, completely independent of method 1, to determine jet source regions from the images. This second approach addresses the problem in a slightly different way (see Figs. 5 and 6). For each image we used the measured angles $\alpha$ of the jets as seen by the OSIRIS camera and compared them with the surface normal vectors of each illuminated facet. We searched for those facet normals that lie within a certain angular tolerance limit (in this case $15^{\circ}$ ), which is the angle between the plane containing the facet normal and the observer line of sight, and the plane containing the given jet angle and the observer line of sight. The latter plane will intersect a line of facets across the nucleus. This was repeated for all detected jets in a given image. We also localized the jet source regions by adding many more images to the analysis. Each facet was then assigned a score according to the amount of times (i.e., the amount of images) that a jet plane intersected that facet. A second angular tolerance limit was used that is related to the minimum angle of the Sun above the local horizon, set here as $10^{\circ}$. The result is a probability map with localized regions giving the maximum likely source regions for the jets. The panels in Fig. 5 show the regions overlaid on the shape model, viewed along the $x$-, $y$-, and $z$-axes (Fig. 6 is a rectangular projection of Fig. 5). Three primary regions are identified (regions A, B, and C), and another small region labeled D (see Fig. 6). A large part of the nucleus surface is not illuminated or contributed very little or nothing to the observed jets. We repeated the entire analysis for the August 2 data set. The regions determined were not as well localized, but are still broadly consistent with the analysis for the July observations. Method 1 is slightly more effective at isolating the source regions at the observational geometry of the August data set. Observational geometry information was computed using the SPICE toolkit (Acton 1996) along with SPICE kernels provided by ESA and kernels provided by the OSIRIS team for rotational phase and shape model orientation. All versions of the shape models used here were determined from OSIRIS images. 

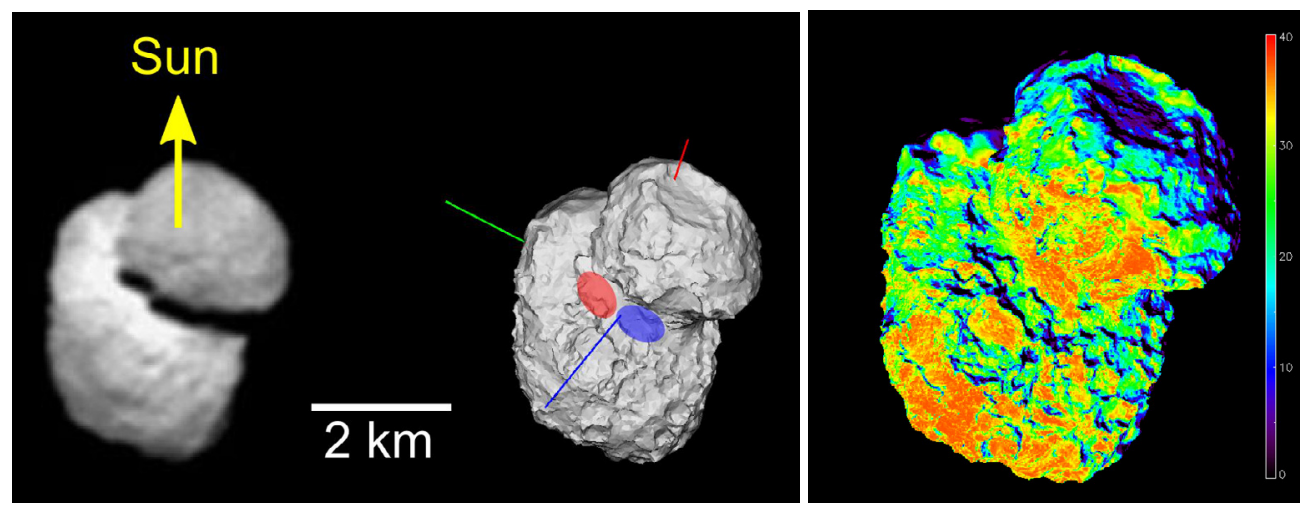

Fig. 4. Results of the inversion technique described in Sect. 3.1. The left image shows a NAC image acquired on 21 July 2014 (spacecraft distance $=4530 \mathrm{~km}$, resolution $=100 \mathrm{~m} / \mathrm{px}$ ). The middle image shows the shape model in the same observing configuration. Red, green, and blue lines indicate the $X, Y$, and $Z$ axes of the model, respectively, with $Z$ being the positive spin axis. The red and blue ellipses mark the locations of jet sources. The right panel displays the solar elevation (in degrees) averaged over the time frame covered by the images obtained on July 20-21. We note that most of the insolation occurs at regions where the detected jet-like features originate from the nucleus, but not all areas with relatively high insolation levels produce a jet source region.

We focused on the $5 \mathrm{k}$-facet version of the shape model (Capanna et al. 2014), which is sufficient for our purposes, although we ran the process on the higher resolution $20 \mathrm{k}$-facet shape for a consistency check, with very similar results (Jorda et al. 2014).

The analysis of images of $67 \mathrm{P}$ acquired by the OSIRIS cameras reported by Tubiana et al. (2015) on April 30, May 4, 2014, and June 24, 2014 also allowed determining the location of active areas on the comet nucleus. During that period of time, the comet moved inbound from $\sim 4.1$ to $\sim 3.8$ AU in heliocentric distance, and a well-developed dust coma showed a fanlike structure toward the north as projected on the plane of the sky. Their simulations with the COSSIM code (Vincent et al. 2010) reproduced the observations when a source (i.e., active area) was located in the northern hemisphere of the comet nucleus at latitudes ranging from $30^{\circ}$ to almost the north pole itself. Comparison of Fig. 10 in Tubiana et al. (2015) with Fig. 6 indicates that the activity has remained at about the same locations since April 2014, that is, mainly the northern hemisphere.

In addition, from WAC images of 67P acquired between August 16 and September 27, 2014, Lin et al. (2015) determined that the jet features detected in the inner coma mostly originated from the Hapi region (see their Fig. 7).

In an attempt to connect these dust features with outgassing from determined regions on the surface and subsurface of the comet nucleus, information provided by VIRTIS (Coradini et al. 2007) on localized gas column densities is very valuable. The VIRTIS instrument started dedicated observations of 67P on 28 July 2014 (at 3.7 AU from the Sun). BockeléeMorvan et al. (2015) concluded that observations carried out from August to September do not show any detection of molecular bands. Only marginal detections of $\mathrm{H}_{2} \mathrm{O}$ and $\mathrm{CO}_{2}$ were obtained in October 2014. The analysis by Bockelée-Morvan et al. (2015) focused on coma observations undertaken between 24 November 2014 and 24 January 2015. Although these observations pertain to later dates than those we analyzed, it is worth to mention that higher water column densities are observed for the line of sight above the neck regions, that is, those that broadly coincide with regions $\mathrm{A}$ and $\mathrm{B}$ in Fig. 5, and with those of higher insolation shown in Figs. 4 and 5. Furthermore, BockeléeMorvan et al. (2015) also concluded that $\mathrm{CO}_{2}$ is outgassing from both illuminated and non-illuminated regions. Thus, the dust jets analyzed in this work might well be produced not only by the water ice sublimation, but also by the carbon monoxide and dioxide sublimation from most active areas when the comet was moving inbound at 4.1 to $2.5 \mathrm{AU}$ from the Sun.

On the other hand, theoretical work by Gundlach et al. (2015) has shown that outgassing of $\mathrm{H}_{2} \mathrm{O}$ ice can only explain the dust activity within $2.5 \mathrm{AU}$ (in broad agreement with the findings of gaseous water by VIRTIS, Bockelée-Morvan et al. 2015). At larger heliocentric distances, as at those studied here, the outgassing is driven by $\mathrm{CO}_{2}$ and $\mathrm{CO}$ and the radius of the dust aggregate particles lifted from the surface range from the centimeter to decimeter range (see Fig. 3 in Gundlach et al. 2015). Thus, these dust aggregates most likely are the ones populating the jet-like features and the coma seen in Figs. 1-3.

We defined an outer boundary to the regions identified in Figs. 5 and 6 using a semi-arbitrary approach, with the boundary defined as being where the number of images contributing to the jet region decreases to 6-7 (see Fig. 7). This is sufficient for our purposes. For each region - color-coded red (A), blue (B), green (C), and yellow (D) - we approximated the emerging jet direction to be the average of the surface normal vectors contained within that region, weighted according the angle of the Sun above the horizon of each facet. This seems to reproduce the observed features very well (see Fig. 8 below). Region A is roughly equivalent to the Hapi region as defined by Sierks et al. (2015) and Thomas et al. (2015), but overlapping slightly with the Hathor and Anuket regions; region B corresponds to the Ma' at and Serqet regions; region $\mathrm{C}$ is Ash with some overlap with Aten; and region D mainly corresponds to Aten. It is worth noting that these regions tend to be relatively smooth terrain, either dust-covered or where spectral slopes are much bluer than average.

In Fig. 8 we display selected examples of the projection of jets from the source regions in Figs. 5-7 onto the observing plane of processed OSIRIS images (from the July 25-26, 2014 data set). This was performed as a visual check on which source regions are contributing to the various jets detected. The right-hand panels of Fig. 8 show the projection of the nucleus as seen from Rosetta at the time of observation, with celestial North aligned with the $+y$-axis (solid green lines). The arrows pointing to the defined source regions show the jet directions and are color-coded to indicate the direction of the Sun at that time from that region (cosine of the zenith angle: red $=1$, blue $=0$ ), and therefore the level of solar insolation. The middle panels are the OSIRIS images themselves, processed (i.e., radial 

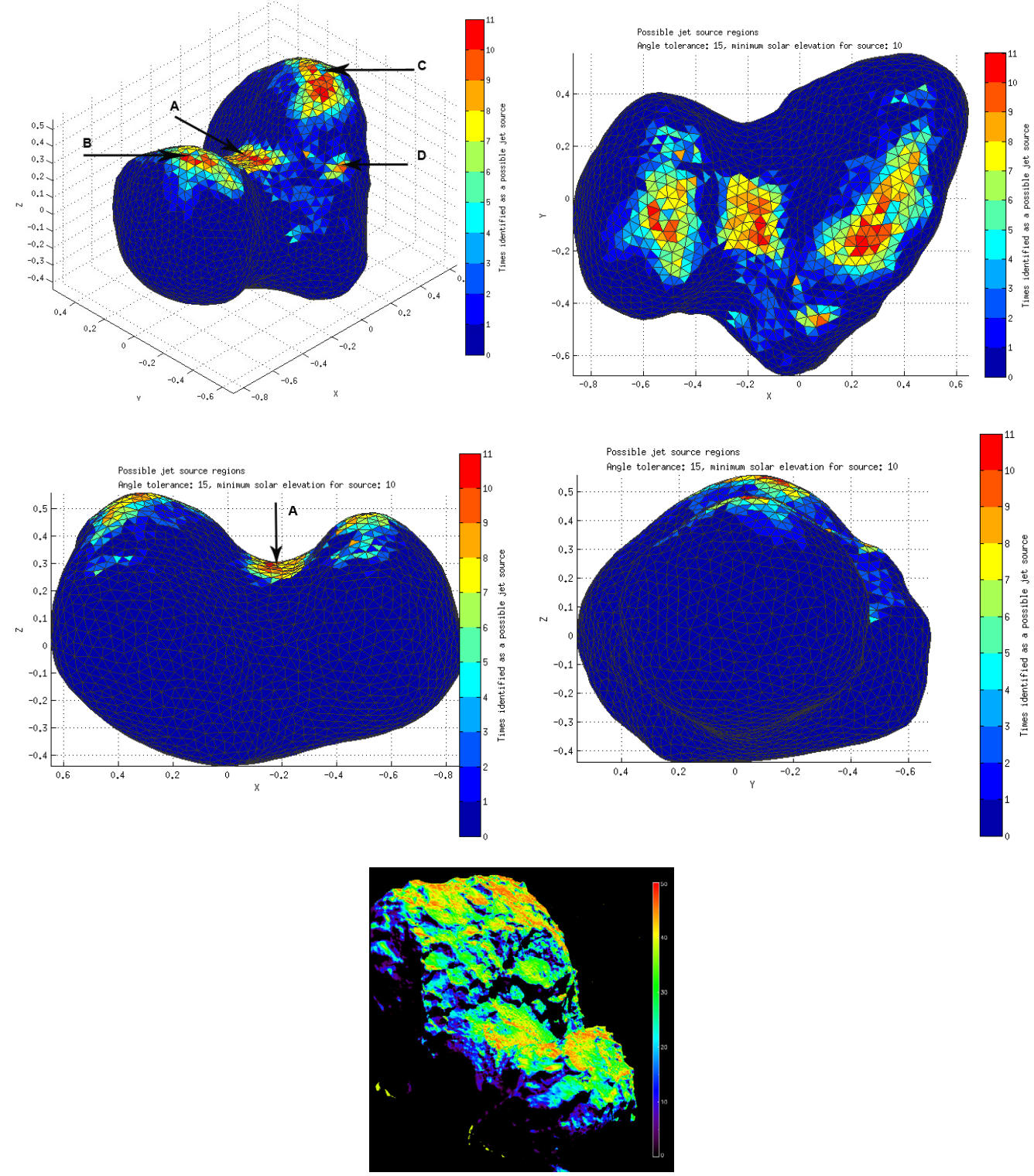

Fig. 5. Nucleus jet-source regions determined using method 2 for the July 25-26 data set. For comparison, the solar elevation (in degrees) averaged over the time frame covered by the above mentioned data set is also shown. We note that most of the insolation occurs at regions where the detected jet-like features originate from the nucleus, but not all areas with relatively high insolation levels produce a jet source region.

renormalization) to enhance the jets, and also rotated to allow direct comparison with the right-hand panels. The solid yellow lines denote the direction of jets detected in OSIRIS images of 67P (whose original location angles are listed in Table 1 measured on non-rotated WAC frames, see also Fig. 2, left panel), the dotted red, blue, yellow, and green lines indicate expected directions of the jets from the corresponding region on the righthand panels. The left-hand panels are included simply to provide a wider aspect on the projected jet directions, at the same size-scale as the images. The top set of panels (corresponding to image 2014-07-25T20:50:54) shows that the projection of jets from regions $\mathrm{A}, \mathrm{B}$, and $\mathrm{C}$, overlap very well with the primary jet detected in the images, located at $\mathrm{PA}=38^{\circ}$ in this image. This is the case for all of the other images in the July 25-26 data set. Jet $\mathrm{D}$ is pointing in a very different direction to the others and does not register a detectable jet in the image. However, as the primary set source regions rotate away and region D is illuminated differently (second set of panels corresponding to image 2014-07-26T02:39:40), a faint second jet appears for which the main source seems to be only region $\mathrm{D}$, given the wide angular separation between $\mathrm{ABC}$ and $\mathrm{D}$. This is repeated on the third and fourth set of panels, corresponding to images 2014-0726T03:18:25 and 2014-07-26T03:57:10. Region D corresponds to the Aten region, whose appearance suggests that large quantities of material may have separated from the comet relatively recently, exposing subsurface ices. The jets from this region are faint, which would tend to indicate that significant surface mantling has occurred since the release (Thomas et al. 2015), but not enough to shut off all activity even at this large heliocentric distance. Small localized icy patches are suggested from surface spectral slope measurements in this region (Fornasier et al. 2015). Spectral mapping from VIRTIS over a wavelength range of $1-2 \mu \mathrm{m}$ also shows localized areas that may indicate the presence of exposed water ice, albeit almost completely insulated with dust (Capaccioni et al. 2015). Moreover, as indicated by Keller et al. (2015), the water erosion integrated over one 


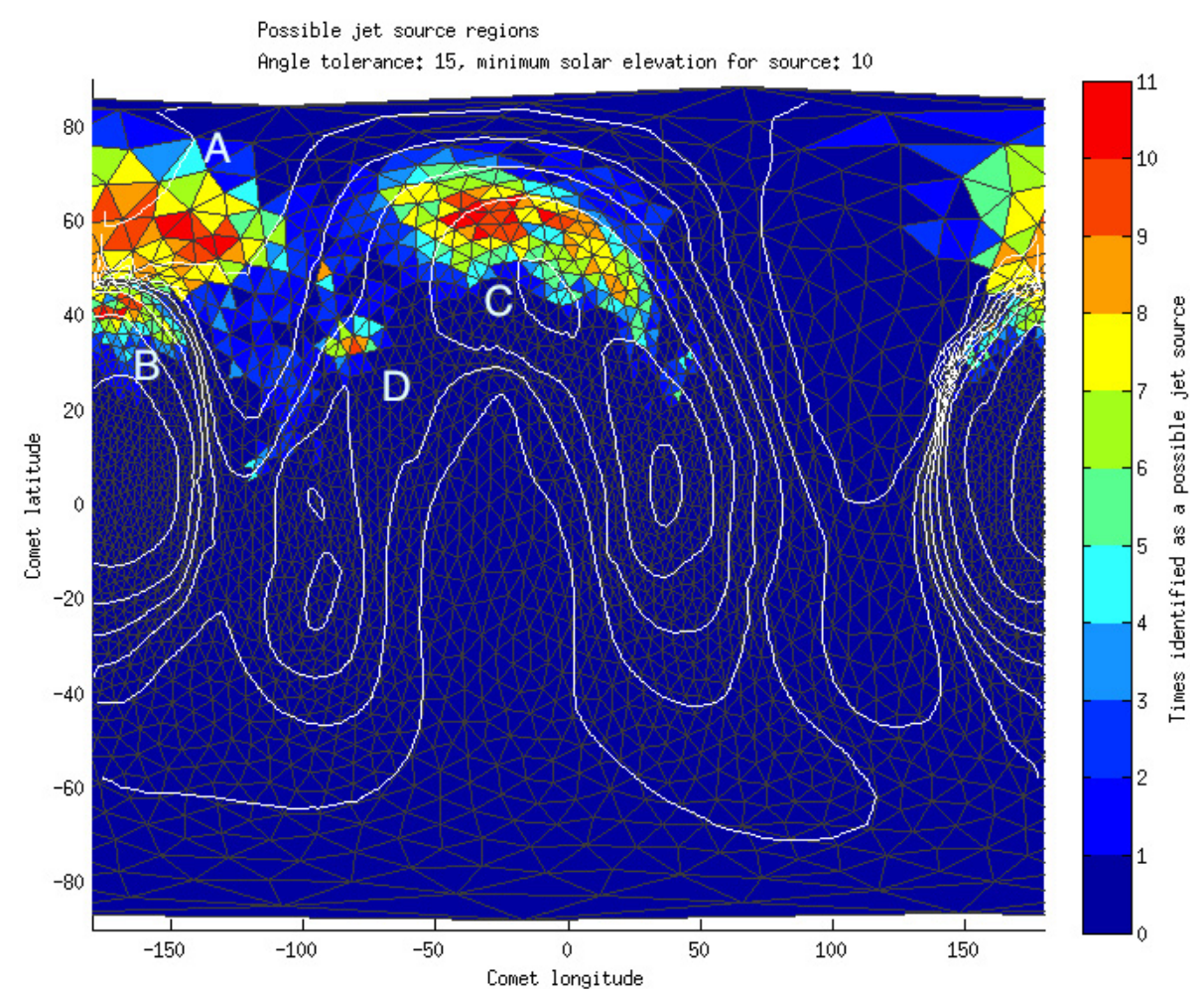

Fig. 6. Rectangular projection of Fig. 5. The contours show the main topographical features of the nucleus. The Hapi region is located near latitude $60^{\circ}$.

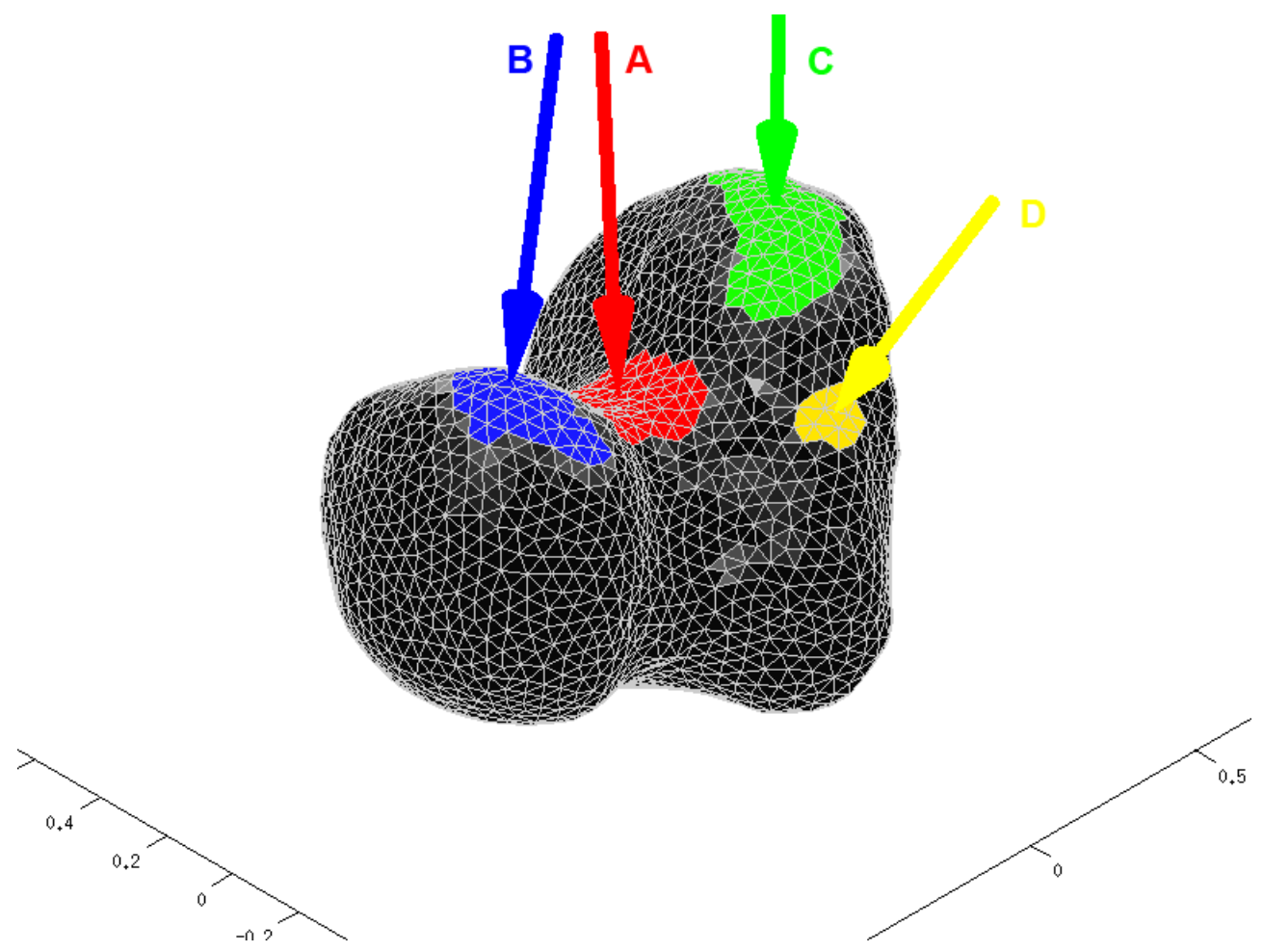

Fig. 7. Defining outer boundaries to jet source regions and approximating jet directions, from method 2. For each region - color-coded red (A), blue (B), green (C), and yellow (D) - we approximate the emerging jet direction to be the average of the surface normal vectors contained with that region, weighted according the angle of the Sun above the horizon of each facet. This seems to reproduce the observed features very well (see Fig. 8).

nucleus rotation on August 9, 2014 is higher on the northern hemisphere and the neck region. The erosion, and thus the activity, is enhanced along the transition from the valley bottom (Hapi) to the cliff wall (Hathor); these regions correspond to the places where the jet-like features originate as deduced from both method 1 and 2 .

\section{Conclusions}

In addition to the reported activity since March 2014 at $4.3 \mathrm{AU}$ (Tubiana et al. 2015) and the activity outburst somewhen between 27 and 30 April 2014 (Mottola et al. 2014), comet 67P shows a clearly non-isotropic dust coma whose appearance to the 
L. M. Lara et al.: 67P dust jets and surface active areas at large heliocentric distance

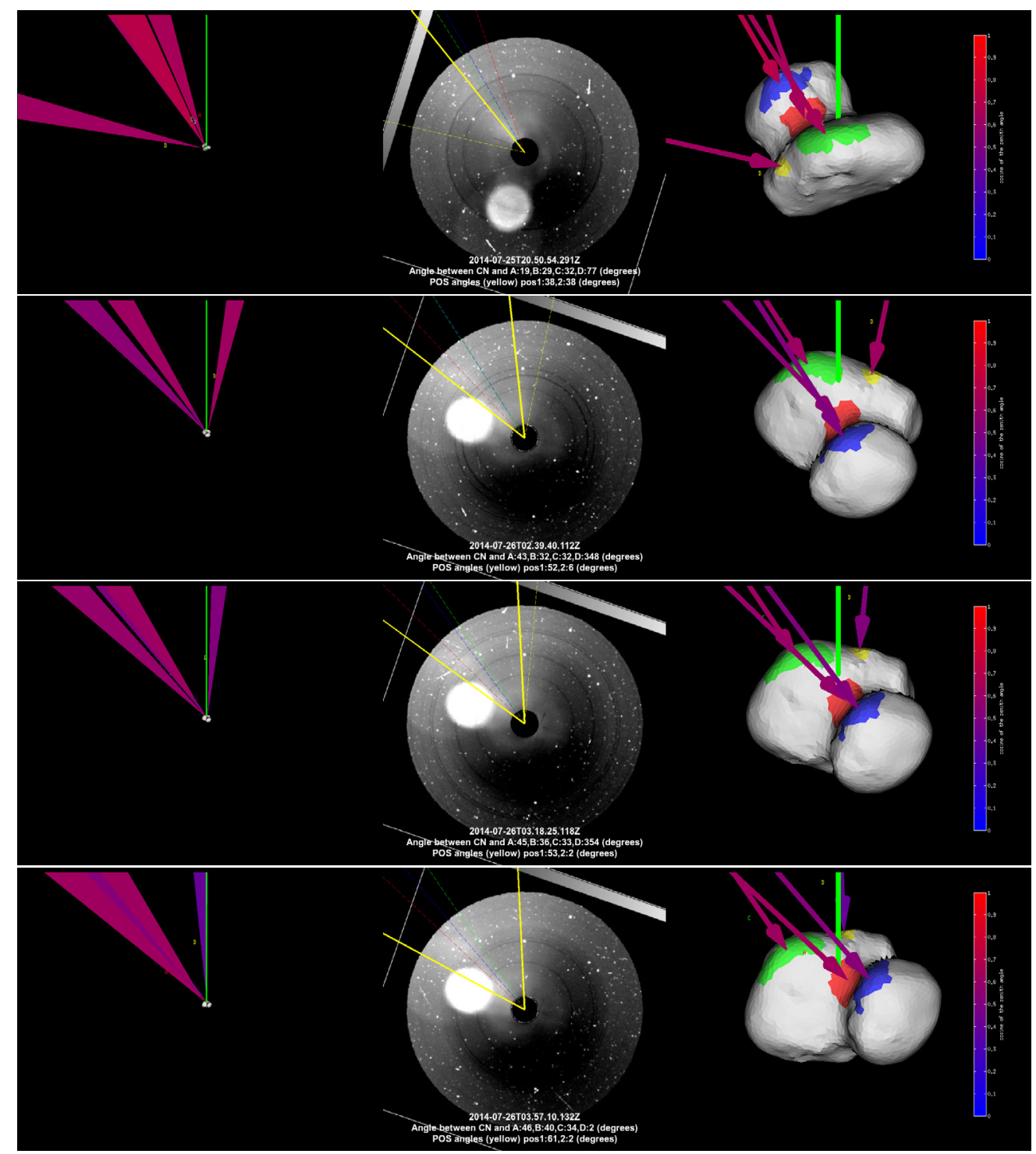

Fig. 8. Selected examples of the projection of jets from the source regions in Figs. 5-7 onto the observing plane of processed OSIRIS images (from the July 25-26, 2014 data set). In the lower three panels we see signs of an additional faint jet from the Aten region of the nucleus. The source region of this jet is shown in yellow in the panels above. See Sect. 3.2 for details.

observer (i.e., to the OSIRIS cameras onboard Rosetta) changes with nucleus rotation and with date. Here we have analyzed three sequences of images acquired in July and August 2014 in which obvious dust jet-like features have been detected. Two different methods, originally developed to analyze ground-based observations of comets showing dust jets, have allowed us to determine the regions where these features originate. The so-called active areas pertain to the Hapi, Hathor, Anuket, Ma'at, Serqet, Ash, and Aten regions, which are very well spatially resolved by the OSIRIS camera. The work presented here validates the models by Vincent et al. (2010, 2013), as they allow the determination of main active areas and their evolution in time.

\section{All of these active regions}

- show distinct geomorphological features, Hapi (together with Imhotep) being the smoothest terrains on the comet surface (at the scale provided by the OSIRIS images);

- Hapi, Hathor and part of Seth and Ma' at have a low spectral slope (Fornasier et al. 2015) and high albedo. Similarly, Anuket and Serqet are slightly redder than Hapi;
- show tentative exposed water ice (Capaccioni et al. 2015); and

- receive more solar insolation and self-illumination (especially at Hapi) and higher temperatures along the foot of the Hathor wall, which causes higher activity levels (BockeléeMorvan et al. 2015) and thus erosion (Keller et al. 2015).

Therefore, although the global comet activity is driven by solar insolation, there are some distinct areas where this activity is enhanced and gas and dust is emitted, such as large-scale dust jets. Comets Wild 2 (Sekanina et al. 2004), 9P/Tempel 1 (Farnham et al. 2007), and 103P/Hartley 2 (A'Hearn et al. 2011) all also clearly had jet-like features that originated from non-illuminated sources. They might be driven by very volatile ices or by thermal lags that drive activity after sun set. A similar behavior (nighttime activity) has not yet been clearly detected from the nucleus of $67 \mathrm{P}$.

As the monitoring of $67 \mathrm{P}$ by the Rosetta spacecraft continues, much higher spatial resolution (on the order of m/pix and below) have provided us with detailed images of the 
regions where the large-scale jets originate. When the OSIRIS cameras have a limb view of these active regions, complex "curtains" of jets can be distinguished against the dark background (i.e., space). These small-scale dust jets can be seen at certain nucleus regions that broadly correspond to the latitudes derived in this work in the Hapi, Hathor, Anuket, Ma'at, Serqet, Ash, and Aten regions. During August and September, jet-like features continued to be detected and were analyzed in Lin et al. (2015). Very recently, Spitale et al. (2015) have shown that in the case of Enceladus, the eruptive activity can be explained by broad, curtain-like eruptions where a combination of viewing direction and local fracture geometry produce individual image features looking like discrete jets. Thus, for the case of 67P, special care has to be taken when attempting to connect jet-like features with discrete regions as long fractures might be at the origin of these features.

Unfortunately, since the spacecraft entered into orbit around 67P on August 5, 2014 to the present day, there have not been spacecraft excursions at large distances (several hundreds of $\mathrm{km}$ ) to have a wider view of the dust coma now that hundreds of small jets are clearly identified (at least in terms of angular size in the coma). This would help to attempt a physical connection between tiny-to-small and large-scale jets seen since mid-July 2014, a question that still remains open. The analysis shown here and the much higher spatial resolution seem to point to a very simplistic conclusion: large-scale jets seen by any observer at large distances might likely be due to the contribution of many small jets. The physical processes (i.e., dynamics) that cause the connection between these two scales need further modeling, which is beyond the scope of this paper, however.

At this point, we would like to emphasize that as the nucleus approaches perihelion (at 1.24 AU on August 13, 2015), the gas and dust emission pattern from equatorial and mid-latitudes will be such that the large-scale jets seen from the ground during 2003 (Weiler et al. 2004) and 2009 (Lara et al. 2011; Vincent et al. 2013) will be spatially resolved on the nucleus into many small jets or, in contrast, into a wide active area producing single jets. The Rosetta spacecraft will fly a series of close and far fly-bys ranging from $15 \mathrm{~km}$ (strict minimum spacecraft-nucleus distance) up to $1500 \mathrm{~km}$. These will permit an integrated view of the dust jets in the very inner and outer coma of 67P.

Acknowledgements. OSIRIS was built by a consortium led by the MaxPlanck-Institut für Sonnensystemforschung, Göttingen, Germany, in collaboration with CISAS, University of Padova, Italy, the Laboratoire d'Astrophysique de Marseille, France, the Instituto de Astrofisica de Andalucia, CSIC, Granada, Spain, the Scientific Support Office of the European Space Agency, Noordwijk, The Netherlands, the Instituto Nacional de Tecnica Aeroespacial, Madrid, Spain, the Universidad Politecnica de Madrid, Spain, the Department of Physics and Astronomy of Uppsala University, Sweden, and the Institut für Datentechnik und Kommunikationsnetze der Technischen Universität Braunschweig, Germany. The support of the national funding agencies of Germany (DLR), France (CNES), Italy (ASI), Spain (MINECO), Sweden (SNSB), and the ESA Technical Directorate is gratefully acknowledged. We thank the Rosetta Science Ground Segment at ESAC, the Rosetta Mission Operations Centre at ESOC and the Rosetta Project at ESTEC for their outstanding work, enabling the science return of the Rosetta Mission. We gratefully acknowledge the developers of SPICE and NAIF/PDS resources. L. M. Lara is very thankful to J. M. Bacaicoa for his assistance with image editing. This work was funded by grant AyA 2012-32237 awarded by the Spanish Ministerio de Economia y Competitividad.

\section{References}

Acton, C. H. 1996, Planet. Space Sci., 44, 65

A'Hearn, M. F., Hoban, S., Birch, P. V., et al. 1986, Nature, 324, 649

A'Hearn, M. F., Belton, M. J. S., Delamere, W. A., et al. 2011, Science, 332, 1396
Bockelée-Morvan, D., Debout, V., Erard, S., et al. 2015, A\&A, 583, A6 Boehnhardt, H., \& Birkle, K. 1994, A\&AS, 107, 101

Capaccioni, F., Coradini, A., Filacchione, G., et al. 2015, Science, 347, 628

Capanna, C., Jorda, L., Gesquière, G., et al. 2014, AGU Fall Meeting Abstracts, C3941

Coradini, A., Capaccioni, F., Drossart, P., et al. 2007, Space Sci. Rev., 128, 529

Farnham, T. L., Wellnitz, D. D., Hampton, D. L., et al. 2007, Icarus, 187, 26

Farnham, T. L., Bodewits, D., Li, J.-Y., et al. 2013, Icarus, 222, 540

Feaga, L. M., A'Hearn, M. F., Sunshine, J. M., Groussin, O., \& Farnham, T. L. 2007, Icarus, 190, 345

Fornasier, S., Hasselmann, P. H., Barucci, M. A., et al. 2015, A\&A, 583, A30

Fulle, M., Barbieri, C., Cremonese, G., et al. 2004, A\&A, 422, 357

Gulkis, S., Allen, M., von Allmen, P., et al. 2015, Science, 347, 709

Gundlach, B., Blum, J., Keller, H., \& Skorov, Y. 2015, A\&A, 583, A12

Hässig, M., Altwegg, K., Balsiger, H., et al. 2015, Science, 347, 276

Jorda, L., Gaskell, R. W., Hviid, S. F., et al. 2014, AGU Fall Meeting Abstracts, C3943

Keller, H. U., Barbieri, C., Lamy, P., et al. 2007, Space Sci. Rev., 128, 433

Keller, H. U., Mottola, S., Davidsson, B., et al. 2015, A\&A, 583, A34

Lara, L. M., Lin, Z.-Y., Rodrigo, R., \& Ip, W.-H. 2011, A\&A, 525, A36

Larson, S. M., \& Sekanina, Z. 1984, AJ, 89, 571

Lin, Z.-Y., Lara, L. M., Vincent, J. B., \& Ip, W.-H. 2012, A\&A, 537, A101

Lin, Z.-Y., Ip, W.-H., Lai, I.-L., et al. 2015, A\&A, 583, A11

Moreno, F., Lara, L. M., Muñoz, O., López-Moreno, J. J., \& Molina, A. 2004, ApJ, 613, 1263

Mottola, S., Lowry, S., Snodgrass, C., et al. 2014, A\&A, 569, L2

Mueller, B. E. A., Samarasinha, N. H., Farnham, T. L., \& A'Hearn, M. F. 2013, Icarus, 222, 799

Preusker, F., Scholten, F., Matz, K.-D., et al. 2015, A\&A, 583, A33

Samarasinha, N. H., \& Larson, S. M. 2014, Icarus, 239, 168

Sekanina, Z., Brownlee, D. E., Economou, T. E., Tuzzolino, A. J., \& Green, S. F. 2004, Science, 304, 1769

Sierks, H., Barbieri, C., Lamy, P. L., et al. 2015, Science, 347, 1044

Spitale, J. N., Hurford, T. A., Rhoden, A. R., Berkson, E. E., \& Platts, S. S. 2015, Nature, 521, 57

Thomas, N., Sierks, H., Barbieri, C., et al. 2015, Science, 347, 440

Tubiana, C., Snodgrass, C., Bertini, I., et al. 2015, A\&A, 573, A62

Vincent, J.-B., Böhnhardt, H., \& Lara, L. M. 2010, A\&A, 512, A60

Vincent, J.-B., Lara, L. M., Tozzi, G. P., Lin, Z.-Y., \& Sierks, H. 2013, A\&A, 549, A 121

Vincent, J.-B., Bodewits, D., Besse, S., et al. 2015, Nature, 523, 63

Weiler, M., Rauer, H., \& Helbert, J. 2004, A\&A, 414, 749

1 Instituto de Astrofisica de Andalucia-CSIC, Glorieta de la Astronomia, 18008 Granada, Spain

e-mail: lara@iaa.csic.es

2 Centre for Astrophysics and Planetary Science, School of Physical Sciences, The University of Kent, Canterbury CT2 7NH, UK

3 Max-Planck Institut fuer Sonnensystemforschung, Justus-vonLiebig-Weg, 337077 Goettingen, Germany

4 Department of Physics and Astronomy G. Galilei, University of Padova, Vic. Osservatorio 3, 35122 Padova, Italy

5 Center of Studies and Activities for Space (CISAS) "G. Colombo", University of Padova, via Venezia 15, 35131 Padova, Italy

6 Aix Marseille Université, CNRS, LAM (Laboratoire d'Astrophysique de Marseille) UMR 7326, 13388, Marseille, France

7 Centro de Astrobiologia (INTA-CSIC), European Space Agency (ESA), European Space Astronomy Centre (ESAC), PO Box 78, 28691 Villanueva de la Canada, Madrid, Spain

8 International Space Science Institute, Hallerstrasse 6, 3012 Bern, Switzerland

9 Research and Scientific Support Department, European Space Agency, 2201 Noordwijk, The Netherlands

10 PAS Space Reserch Center, Bartycka 18A, 00716 Warszawa, Poland

11 Institute for Geophysics and Extraterrestrial Physics, TU Braunschweig, 38106 Braunschweig, Germany

12 Department for Astronomy, University of Maryland, College Park, MD 20742-2421, USA

13 LESIA, Observatoire de Paris, CNRS, UPMC Univ Paris 06, Univ. Paris-Diderot, 5 place J. Janssen, 92195 Meudon Principal Cedex, France 
L. M. Lara et al.: 67P dust jets and surface active areas at large heliocentric distance

14 LATMOS, CNRS/UVSQ/IPSL, 11 boulevard d'Alembert, 78280 Guyancourt, France

15 INAF Osservatorio Astronomico di Padova, vic. dell'Osservatorio 5, 35122 Padova, Italy

16 Department of Physics and Astronomy, Uppsala University, Box 516, 75120 Uppsala, Sweden

${ }_{17}$ CNR-IFN UOS Padova LUXOR, via Trasea 7, 35131 Padova, Italy

18 Department of Industrial Engineering, University of Padova, via Venezia 1, 35131 Padova, Italy

19 University of Trento, via Sommarive 9, 38123 Trento, Italy

20 Physikalisches Institut, Sidlerstrasse 5, University of Bern, 3012 Bern, Switzerland
21 INAF-Osservatorio Astronomico di Trieste, via Tiepolo 11, 34143 Trieste, Italy

22 Deutsches Zentrum für Luft- und Raumfahrt (DLR), Institut für Planetenforschung, Rutherfordstrasse 2, 12489 Berlin, Germany

23 Institute for Space Science, National Central University, 32054 Chung-Li, Taiwan

${ }^{24}$ ESA/ESAC, PO Box 78, 28691 Villanueva de la Cañada, Spain

25 Department of Information Engineering, University of Padova, via Gradenigo 6/B, 35131 Padova, Italy

26 Instituto Nacional de Técnica Aeroespacial, 28850 Torrejón de Ardoz, Madrid, Spain 